\title{
Critical conditions for non-symmetric flame propagation in narrow channels: influence of the flow rate, the thermal expansion, the Lewis number and heat-losses
}

\author{
Anne Dejoan, Carmen Jimenez, Vadim N. Kurdyumov ${ }^{1}$ \\ Department of Energy, CIEMAT, Madrid, Spain
}

\begin{abstract}
This paper presents a numerical study of the propagation of isobaric premixed flames in narrow channels, focusing primarily on the symmetry of solutions. Because of the complexity of the problem, only few but very important effects such as the thermal expansion, the Lewis number and heat losses are included in the formulation and only a planar channel geometry is investigated. It is demonstrated that symmetric and non-symmetric steady state flames can exist simultaneously, for certain ranges of the parameters.

The parametric dependence of the corresponding bifurcation points is also investigated. It is found that both subcritical and supercritical bifurcations are possible for different values of the parameters. The steady-state results are successfully compared with direct timedependent simulations. The simulations show that, as a general rule, in cases where both types of solutions exist simultaneously, the non-symmetric solutions are stable while the symmetric solutions are unstable.
\end{abstract}

\section{Introduction}

The flame propagation in ducts and channels of different cross-sections represents a cornerstone problem in combustion science with multiple applications in many practical combustion devices. The main complication arising in the study of this problem resides in the fact that various physical phenomena act simultaneously in the process, thus creating serious obstacles in getting a clear physical insight. The properties of the combusting mixture (reactivity / heat release / diffusivity), the rate at which it flows in the duct, the geometry of this duct or the heat transfer to and from the walls all intervene in determining the characteristics of the propagating flame.

After pioneering works on the numerical analysis of flames propagating in ducts $[1,2]$, the interest in small-scale combustion systems has recently revived the studies on the effects of the channel flow rate $[3,4]$, heat-losses [5, 14], the Lewis number $[4,6,7,13,15-17]$ and thermal expansion [8-12,18-21] on the propagation of flames in narrow channels.

\footnotetext{
${ }^{1}$ Corresponding author
} 
In many of the above mentioned works it was assumed from the start that the flame shape should be symmetric with respect to a line of symmetry (axisymmetric in circular channels or symmetric about the midplane in two-dimensional channels) $[1-3,5-7,9,10,12,31]$. This restriction on symmetry for freely propagating flames was removed systematically in $[4,11]$ and the issue has received a significant attention only recently, see [13-17, 20, 21]. Experimental observations of non-symmetric premixed flames freely propagating in narrow channels appeared in [22], for example.

The results presented in [13] showed that in narrow channels, for mixtures with Lewis number smaller than one, symmetric and non-symmetric solutions may co-exist for the same set of parameters. It was demonstrated by means of global stability analysis that when this is the case, the non-symmetric solution is stable while the symmetric solution is usually unstable. This effect of symmetry breaking was first demonstrated in the context of a constant density (diffusive-thermal) model and using a global one-step Arrhenius kinetics with a single reactant characterized by a constant Lewis number, so that it could be unmistakably linked to the diffusive-thermal instability [4, 13-16]. A similar effect was reported in [11], based on the complete Navier-Stokes equations in isothermal and appreciably wider ducts. These results have been validated recently within a more realistic direct simulation model including the detailed chemical kinetics and transport for lean hydrogen-air flames propagating in adiabatic channels and channels with heat losses [20, 21].

The possibility of symmetry breaking for flames with Lewis number larger than one was illustrated in [13] in a case with $L e=4$, showing non-symmetric oscillatory dynamics during flame propagation. Even though such high values of the Lewis number can be found, for example, in a mixture of butane and oxygen diluted with helium [23], these cases are not considered in the present study, focusing attention on Lewis number variations not far from unity.

It should be mentioned that the existence of non-symmetric flames was also reported not long ago for completely different configurations. In these experimental [24] and numerical [25-28] investigations, instead of freely propagating, the flames were stabilized by means of partially heated walls in narrow conducts. The symmetry breaking under these conditions is not necessarily related to Lewis number or thermal expansion effects, as shown in [29], where it was demonstrated for $L e=1$ within the constant density model. It must be remarked that, even if being dynamically unsteady in the above configurations, the flame of this kind does not show free propagation, and is therefore different from the flames explored in the present study.

The present work investigates the combined influence of the thermal expansion and the differential diffusion effects on breaking the flame symmetry for flames freely propagating in narrow channels, using a one-step Arrhenius kinetics and a zero-Mach-number approximation. We 
include also in the study the effects of the reactants flow rate and heat losses through the channel walls. Undoubtedly this leaves aside some important effects that have received attention recently, such as the influence of buoyancy forces acting in horizontal or vertical channel configurations studied in [30]. An important part of the present results is related to the determination of the critical flash-back points, namely, the conditions for which the flame is stabilized with respect to the channel wall. This state was under consideration recently in [31] where only symmetric solutions were considered.

The numerical costs of studying the flame symmetry breaking in planar and circular channels are clearly different. Because the last case requires vastly more numerical resources, only a planar 2D configuration is treated in the present work. Perhaps this simplification could mean that some important effects related, for example, to an additional flame curvature appearing in circular channels are overlooked. Nevertheless we expect that the main trends reported in the present study will persist in circular-channel geometry. The corresponding 3D results will be reported elsewhere.

The aim of the current study is not to analyze exhaustively the full range of parameters and their possible combinations but rather to investigate tendencies leading to the emergence of nonsymmetric flames in narrow channels. In particular we focus on the estimation of the critical conditions for which the symmetry breaking takes place and the influence of this symmetry breaking on the critical flash-back conditions.

The article is arranged as follows: in Section 2, we present the general formulation; the numerical treatment is briefly described in Section 3; numerical results describing steady-state flames are outlined in Section 4 and the results of time-dependent modeling are given in Section 5. Finally, conclusions are drawn in the last section.

\section{General formulation}

Consider a combustible mixture (fuel and oxidizer) at initial temperature $T_{0}$, density $\rho_{0}$ and fuel mass fraction $Y_{0}$, flowing in a channel with mean velocity $U_{0}$. For the sake of simplicity, we consider planar two-dimensional channels. In what follows, $h$ and $h_{w}$ denote the channel height and the wall thickness, respectively, and $x^{\prime}, y^{\prime}$ denote the longitudinal and wall-normal coordinates. Primes here and hereafter mark dimensional quantities if the same notation is used for dimensional and non-dimensional variables. The subindex " 0 " indicates initial fresh stream values.

It is assumed that the external surface of the wall is maintained at the temperature of the 
fresh mixture $T_{0}$. If the wall is thin compared with the channel width, $h_{w} \ll h$, the temperature distribution inside the wall is linear. Using continuity of the heat flux at $y^{\prime}=0$ and $y^{\prime}=h$, the temperature boundary conditions at the internal wall surfaces become

$$
\left.\lambda_{g} \frac{\partial T}{\partial y^{\prime}}\right|_{y^{\prime}=0}=\frac{\lambda_{w}}{h_{w}}\left(\left.T\right|_{y^{\prime}=0}-T_{0}\right), \quad-\left.\lambda_{g} \frac{\partial T}{\partial y^{\prime}}\right|_{y^{\prime}=h}=\frac{\lambda_{w}}{h_{w}}\left(\left.T\right|_{y^{\prime}=h}-T_{0}\right),
$$

where $\lambda_{g}$ and $\lambda_{w}$ are the thermal conductivities of the gas and the wall material, respectively.

The combustible mixture undergoes a chemical reaction modeled by a global irreversible step $F+O \rightarrow P$, where $F$ denotes the fuel, $O$ the oxidizer and $P$ the products. Assuming that the mixture is lean in fuel, the oxidizer mass fraction remains nearly constant. The amount of fuel consumed per unit volume and per unit time is given by $\Omega=\mathcal{B} \rho^{n} Y \exp \left(-E / \mathcal{R}_{g} T\right)$, where $\mathcal{B}$ is a pre-exponential factor, $\rho$ is the density of the mixture, $Y$ is the fuel mass fraction, $E$ is the overall activation energy, and $\mathcal{R}_{g}$ is the universal gas constant. There is no unanimous agreement in the combustion literature concerning the power $n$, and values $n=1$ and $n=2$ have been used in different studies. We assume also constant transport properties and heat capacity $c_{p}$ of the mixture.

The burning velocity of the planar flame $S_{L}$, the thermal flame thickness defined as $\delta_{T}=$ $\mathcal{D}_{T} / S_{L}$, with $\mathcal{D}_{T}=\lambda_{g} / \rho_{0} c_{p}$ the thermal diffusivity, and the adiabatic flame temperature $T_{a}=$ $T_{0}+Q Y_{0} / c_{p}$, with $Q$ the total heat of combustion per unit mass of fuel, are used below to specify the non-dimensional parameters. Using the above scales based on the flame properties allows to compare numerical results calculated for different chemical and transport parameters. To this end, the dimensionless channel width, $a=h / \delta_{T}$, and the dimensionless flow rate, $m=U_{0} / S_{L}$, are introduced. ${ }^{2}$

Dimensionless variables are defined as follows

$$
\begin{gathered}
x=x^{\prime} / \delta_{T}, \quad y=y^{\prime} / h, \quad u=u^{\prime} / S_{L}, \quad v=v^{\prime} /\left(a S_{L}\right), \quad \rho=\rho^{\prime} / \rho_{0}, \\
Y=Y^{\prime} / Y_{0}, \quad \theta=\left(T-T_{0}\right) /\left(T_{a}-T_{0}\right), \quad p=a^{2} p^{\prime} / \rho_{0} S_{L}^{2},
\end{gathered}
$$

where $u, v$ are the velocity components in the $x$ - and $y$-directions, respectively, and $p$ is the pressure.

Two types of calculations were carried out in the present study. For the first type, used to calculate the steady-state solutions (thus imposing $\partial / \partial t=0$ in the governing equations), the reference frame was attached to the flame, namely to a point $\left(x_{*}, y_{*}\right)$ with a given temperature $\theta=\theta_{*}$. The velocity of this point with respect to the wall, $u_{f}=U_{f} / S_{L}$, constitutes an eigenvalue of the problem and gives the flame propagation velocity. Indeed, in the case of steady flame

\footnotetext{
${ }^{2}$ Notice that instead of $a$, the reduced Damköhler number $d=\left(h / \delta_{T}\right)^{2}$ was used in [13, 14] in order to measure the channel width.
} 
propagation the whole flame surface propagates with a constant velocity equal to $u_{f}$, which is independent of both the location of the reference point and of the reference temperature. Evidently, $\theta_{*}$ and $y_{*}$ must be chosen judiciously so that this point lies outside the quenching layer near the wall (if it exists). The calculations of the second type were carried out to investigate the time-dependent flame dynamics, and in this case a reference frame attached to the wall was used. In this case $u_{f}=0$ was imposed in the governing equations presented below. Notice that in the two types of calculations $u$ and $v$ represent the flow velocities with respect to the wall.

Under the above and zero-Mach number assumptions, the governing equations and the equation of state are

$$
\begin{gathered}
\frac{\partial \rho}{\partial t}+\frac{\partial\left(\rho\left[u+u_{f}\right]\right)}{\partial x}+\frac{\partial(\rho v)}{\partial y}=0 \\
\rho\left(\frac{\partial u}{\partial t}+\left[u+u_{f}\right] \frac{\partial u}{\partial x}+v \frac{\partial u}{\partial y}\right)=-\frac{1}{a^{2}} \frac{\partial p}{\partial x}+\operatorname{Pr}\left[\frac{1}{a^{2}} \frac{\partial^{2} u}{\partial y^{2}}+\frac{4}{3} \frac{\partial^{2} u}{\partial x^{2}}+\frac{1}{3} \frac{\partial^{2} v}{\partial x \partial y}\right] \\
\rho\left(\frac{\partial v}{\partial t}+\left[u+u_{f}\right] \frac{\partial v}{\partial x}+v \frac{\partial v}{\partial y}\right)=-\frac{1}{a^{4}} \frac{\partial p}{\partial y}+\operatorname{Pr}\left[\frac{1}{a^{2}}\left(\frac{4}{3} \frac{\partial^{2} v}{\partial y^{2}}+\frac{1}{3} \frac{\partial^{2} u}{\partial x \partial y}\right)+\frac{\partial^{2} v}{\partial x^{2}}\right] \\
\rho\left(\frac{\partial Y}{\partial t}+\left[u+u_{f}\right] \frac{\partial Y}{\partial x}+v \frac{\partial Y}{\partial y}\right)=\frac{1}{L e}\left(\frac{\partial^{2} Y}{\partial x^{2}}+\frac{1}{a^{2}} \frac{\partial^{2} Y}{\partial y^{2}}\right)-\omega \\
\rho\left(\frac{\partial \theta}{\partial t}+\left[u+u_{f}\right] \frac{\partial \theta}{\partial x}+v \frac{\partial \theta}{\partial y}\right)=\left(\frac{\partial^{2} \theta}{\partial x^{2}}+\frac{1}{a^{2}} \frac{\partial^{2} \theta}{\partial y^{2}}\right)+\omega \\
1=\rho(1+q \theta)
\end{gathered}
$$

where $q=\left(T_{a}-T_{0}\right) / T_{0}=Q Y_{0} / c_{p} T_{0}$ is the heat release parameter, $\operatorname{Pr}=\mu c_{p} / \lambda_{g}$ is the Prandtl number, with $\mu$ the viscosity of the mixture and $L e=\lambda_{g} / \rho_{0} c_{p} \mathcal{D}$ is the Lewis number, with $\mathcal{D}$ the mass (fuel) diffusivity. The chemical reaction rate takes the form

$$
\omega=\frac{\beta^{2}}{2 L e u_{p}^{2}}(1+q)^{n} \rho^{n} Y \exp \left\{\frac{\beta(\theta-1)}{1+q(\theta-1) /(1+q)}\right\}
$$

where $\beta=E\left(T_{a}-T_{0}\right) / \mathcal{R}_{g} T_{a}^{2}$ is the Zel'dovich number. In what follows, the values $\operatorname{Pr}=0.7$ and $\beta=10$ were fixed in all cases presented. Anticipating the numerical results presented in the following sections, it was found that the parameter $n$ has little quantitative consequence on the phenomena investigated. For this reason, most of the calculations were carried out for $n=2$ but a comparison with $n=1$ will be given in some figures.

The dimensionless version of Eq. (1), and the no-flux and no-slip conditions at the wall, take the form

$$
\left.\left(\frac{\partial \theta}{\partial y}-b a \theta\right)\right|_{y=0}=\left.\left(\frac{\partial \theta}{\partial y}+b a \theta\right)\right|_{y=1}=\left.\frac{\partial Y}{\partial y}\right|_{y=0,1}=\left.u\right|_{y=0,1}=\left.v\right|_{y=0,1}=0
$$


where $b=\left(\lambda_{w} / \lambda_{g}\right)\left(\delta_{T} / h_{w}\right)$ is the heat transfer parameter. The limiting cases of adiabatic and isothermal walls correspond to $b \rightarrow 0$ and $b \rightarrow \infty$, respectively.

Since axial diffusion becomes negligible far upstream the flame, the state of the gas is constant and uniform with $v=0$, implying that the flow field is unidirectional and satisfies the equations $\partial p / \partial y=0$ and $\partial p / \partial x=\operatorname{Pr} \partial^{2} u / \partial y^{2}$. As a result, a Poiseuille flow is established with

$$
u=6 m y(1-y), \quad v=0, \quad \theta=0, \quad Y=1 \quad \text { as } \quad x \rightarrow-\infty,
$$

where $m=U_{0} / S_{L}$ is the non-dimensional mean flow rate. For far downstream boundary condition we require

$$
\partial^{2} \theta / \partial x^{2}=\partial^{2} Y / \partial x^{2}=\partial u / \partial x=v=0 \quad \text { as } \quad x \rightarrow+\infty .
$$

This mild outlet boundary condition for the temperature field replaces a more severe zerotemperature condition which should be imposed far downstream when heat losses are not negligible. The numerical simulations reported below show that the influence of the downstream boundary condition becomes negligible, as it should be, if the size of the computational domain is reasonably long downstream the flame.

The factor $u_{p}=S_{L} / S_{L}^{a s}$ included in Eq. (9) ensures that the non-dimensional speed of a planar adiabatic flame equals one for a given finite $\beta$, where $S_{L}^{a s}$ is the asymptotic value of adiabatic laminar flame speed calculated at $\beta \rightarrow \infty$ :

$$
S_{L}^{a s}=\sqrt{2\left(\lambda_{0} / c_{p}\right) L e \beta^{-2} \mathcal{B} \rho_{0}^{n-2}\left(T_{0} / T_{a}\right)^{n}} \exp \left(-E / 2 \mathcal{R} T_{a}\right)
$$

Precise calculation of $u_{p}$ requires the solution of the following eigenvalue problem

$$
\begin{aligned}
& d \theta / d z=d^{2} \theta / d z^{2}+\omega, \quad d Y / d z=L e^{-1} d^{2} Y / d z^{2}-\omega, \\
& z \rightarrow-\infty: \quad \theta=Y-1=0, \quad z \rightarrow+\infty: \quad \theta-1=Y=0,
\end{aligned}
$$

where $\omega$ is given by Eq. (9). The numerical values of $u_{p}$ calculated by a shooting method are given in Table 1 for some selected cases.

\section{Numerical treatment}

Steady-state computations were carried out in a finite domain, $x_{\min } \leq x \leq x_{\max }$, using $x_{\min }=-20$ and $x_{\max }=20$ as typical values. All the numerical results reported below were obtained using second-order, three-points central differences for spatial derivatives on a rectangular uniform grid. Typical values for the non-dimensional reference temperature were $\theta_{*}=0.5 \div 0.7$. 


\begin{tabular}{|l|l|c|c|c|c|c|}
\hline & $q \backslash L e$ & 1.05 & 1.0 & 0.9 & 0.8 & 0.7 \\
\hline \multirow{3}{*}{$n=2$} & 5 & 1.0498692 & 1.0546892 & 1.0645288 & 1.0746449 & 1.0850506 \\
& 4 & 1.0539540 & 1.0588333 & 1.0687959 & 1.0790412 & 1.0895828 \\
& 3 & 1.0602406 & 1.0652127 & 1.0753679 & 1.0858156 & 1.0965703 \\
\hline$n=1$ & 5 & 0.9731785 & 0.9773721 & 0.9859223 & 0.9946979 & 1.0037088 \\
\hline
\end{tabular}

Table 1: Values of $u_{p}$ calculated for various $L e$ and $q$, all for $\beta=10$.

The reference point was located at $y_{*} \approx 0.5 \div 0.9$ and approximately in the middle of the domain along the $x$-axis. The independence of the results to these values was verified specifically.

The stream function-vorticity formulation was adopted for the steady-state computations. With the stream function $\psi$, defined from

$$
\rho\left(u+u_{f}\right)=\partial \psi / \partial y, \quad \rho v=-\partial \psi / \partial x
$$

and the vorticity $\zeta=a^{2} \partial v / \partial x-\partial u / \partial y$, the Navier-Stokes equations reduce to

$$
\begin{gathered}
\rho\left(u+u_{f}\right) \frac{\partial \zeta}{\partial x}+\rho v \frac{\partial \zeta}{\partial y}=\operatorname{Pr}\left[\frac{\partial^{2} \zeta}{\partial x^{2}}+\frac{1}{a^{2}} \frac{\partial^{2} \zeta}{\partial y^{2}}\right]+J \\
a^{2} \frac{\partial}{\partial x}\left(\frac{1}{\rho} \frac{\partial \psi}{\partial x}\right)+\frac{\partial}{\partial y}\left(\frac{1}{\rho} \frac{\partial \psi}{\partial y}\right)=-\zeta
\end{gathered}
$$

where $J$ is the vorticity production given by

$$
J=\frac{\partial\left[\rho\left(u+u_{f}\right)\right]}{\partial y} \frac{\partial u}{\partial x}-a^{2} \frac{\partial\left[\rho\left(u+u_{f}\right)\right]}{\partial x} \frac{\partial v}{\partial x}+\frac{\partial(\rho v)}{\partial y} \frac{\partial u}{\partial y}-a^{2} \frac{\partial(\rho v)}{\partial x} \frac{\partial v}{\partial y} .
$$

Equations (16) and (17) along with (6) and (7) and the boundary conditions (properly rewritten in terms of $\psi$ and $\zeta$ ) were solved numerically using a Gauss-Seidel iteration method with successive over-relaxation to determine the eigenvalue $u_{f}$.

In a similar manner to that reported in $[13,14]$, anticipating that the symmetric steady solutions might be unstable, symmetric flames were calculated separately, in half the domain $(0 \leq y \leq 1 / 2)$ and imposing symmetric boundary conditions for the temperature, the mass fraction and the velocities at the centerline, namely

$$
y=1 / 2: \quad \partial \theta / \partial y=\partial Y / \partial y=\partial u / \partial y=v=0 .
$$

In cases where the calculations were carried out in the entire domain, $0 \leq y \leq 1$, the following integral

$$
S=\int_{-\infty}^{\infty} d x \int_{0}^{1 / 2}[\theta(x, y, t)-\theta(x, 1-y, t)] d y
$$


helps to identify the symmetry of the solutions. Clearly, $S=0$ (within numerical accuracy) for a symmetric solution, but it takes a nonzero value when the solution becomes non-symmetric. It should be emphasized that in cases when symmetric solutions exist exclusively, the calculations in the entire domain $(0 \leq y \leq 1 / 2$ led to symmetric solutions (with $S \approx 0$ ).

For all unsteady calculations presented below, the set of dimensionless time-dependent equations (3)-(8) was resolved (imposing $u_{f}=0$ ) making use of the open source code OpenFoam [32]. The implemented numerical method was based on the finite volume method formulated in a collocated grid arrangement. The first order Euler scheme was used for temporal discretization and a second-order scheme for spatial discretization. The flame was maintained in the computational domain using the same methodology as in [17, 20, 21].

\section{Steady-state solutions}

\subsection{Influence of the channel width}

The limit $a=h / \delta_{T} \rightarrow 0$, or the narrow channel approximation, can be easily executed from Eqs. (3)-(7) for the adiabatic case, $b=0$, see [18]. For this purpose all variables are expanded in power series of $a^{2}$, namely $f=f_{0}+a^{2} f_{1}+\ldots$, where $f$ stands for a generic variable. To a first approximation, the resulting equations, implying that the temperature and mass fraction are both function of $x$ only, are identical to those describing the planar flame front, where $u_{f}+m$ represents the flame propagation speed. Thus, by virtue of $u_{p}$ introduced in Eq. (9), $u_{f}+m=1$.

In the presence of heat-losses, for $b>0$, even small, the limit $a \rightarrow 0$ can not be executed for a fixed value of $b$. This follows from Eq. (10) where $a$ appears to the power one thus invalidating the above $a^{2}$-expansion. As a consequence

for a fixed $b>0$ a quenching channel width exists, below which there is no solution. This circumstance can be overcome by introducing the parameter $\tilde{b}=b / a=\left(\lambda_{w} \lambda_{g}\right)\left(\delta_{T}^{2} / h_{w} h\right)$ in the temperature boundary conditions and taking the limit $a \rightarrow 0$ for $\tilde{b}$ fixed. Once again the solution is independent of the $y$-coordinate and, thus, symmetric in this limit. This indicates that there exists a threshold value of the channel width, $a=a_{c}$, above which non-symmetric solutions can exist and below which only symmetric solutions appear.

Consider first the flames with $L e=1$ in adiabatic channels, $b=0$. The dimensionless velocity $u_{f}=U_{f} / S_{L}$ is shown in Fig. 1 (left plot) as a function of the non-dimensional flow rate $m=U_{0} / S_{L}$, calculated for $\beta=10$ and $q=5$ for several values of the channel width, $a=h / \delta_{T}$. The right plot of Fig. 1 shows the symmetry indicator $S$ plotted versus $m$ for the same values of $a$. Solid-line curves correspond to symmetric flames calculated in a half-width 

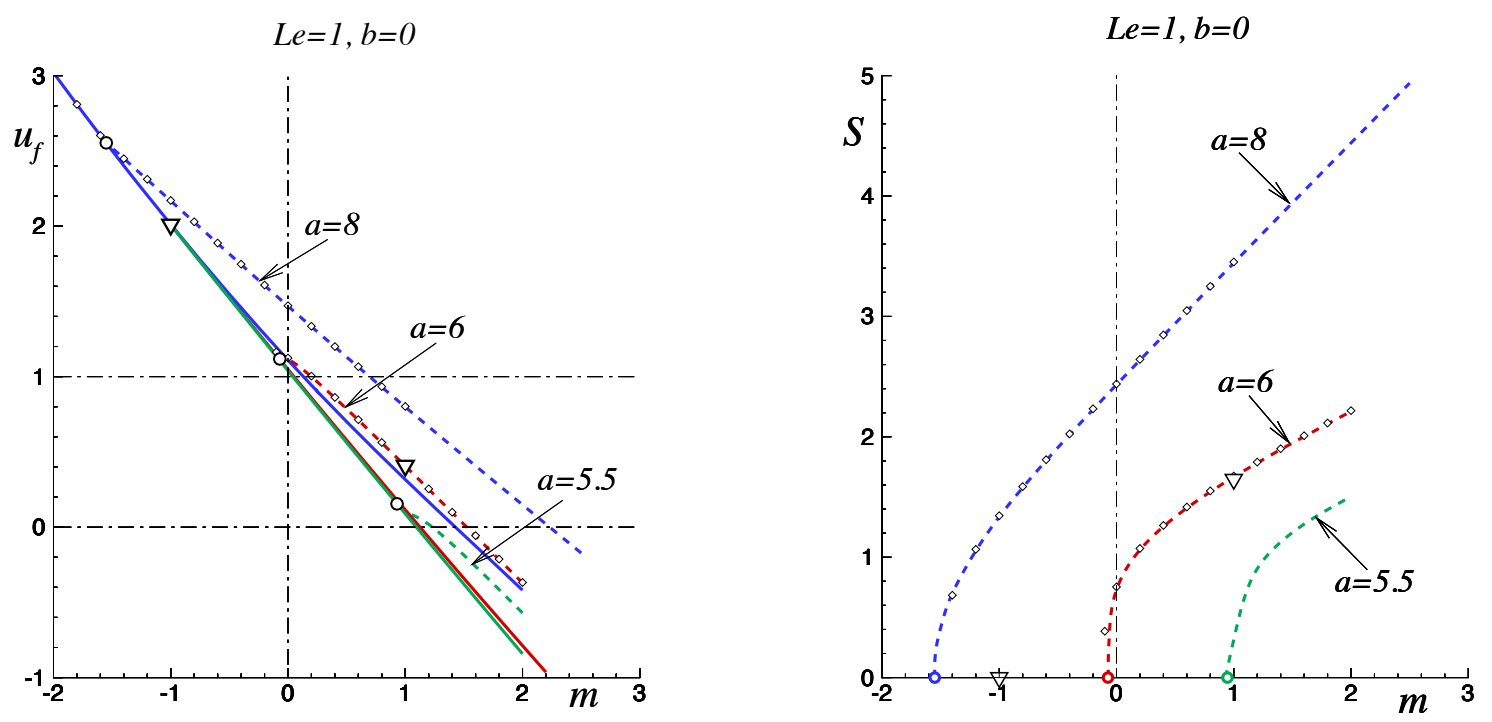

Figure 1: The computed flame velocity $u_{f}=U_{f} / S_{L}$ (left plot) and the symmetry indicator $S$ (right plot) as functions of the non-dimensional flow rate $m=U_{0} / S_{L}$ for $L e=1$ and several values of $a$, all results for $\beta=10, q=5$ and an adiabatic channel, $b=0$. Solid and dashed lines represent symmetric and non-symmetric flames, respectively, calculated with $n=2$; diamond symbols correspond to the cases with $n=1$ (only for $a=6$ and 8); the bifurcation points are marked with open circles. Open gradient symbols represent the results of unsteady calculations for $m=1$ and $m=-1$, both for $a=6$.

domain imposing the symmetric boundary conditions given by Eq. (18) for $n=2$. Dashed-line curves correspond to non-symmetric flames, also for $n=2$. Diamond symbols represent the numerical results calculated with $n=1$.

One can see in Fig. 1 that the parameter $n$ appearing in the reaction rate Eq. (9) has little consequence on the flame velocity $u_{f}$ and the symmetry indicator $S$, thus affecting only slightly the bifurcation value of the flow rate, at least in the considered range of parameters. For this reason the majority of calculations presented below were carried out for $n=2$. Open triangles in this figure show the results of unsteady calculations with $n=2$, used to validate the numerical codes and to verify the stability of the solutions, as will be discussed in Section 5.

Two typical solutions are shown in Fig. 2. These flames were obtained for the same set of parameters ( $a=6$ and $m=1$ ) where the upper solution is symmetric and the other one is non-symmetric.

Figure 1 illustrates the impact of channel width on the emergence of non-symmetric solutions 

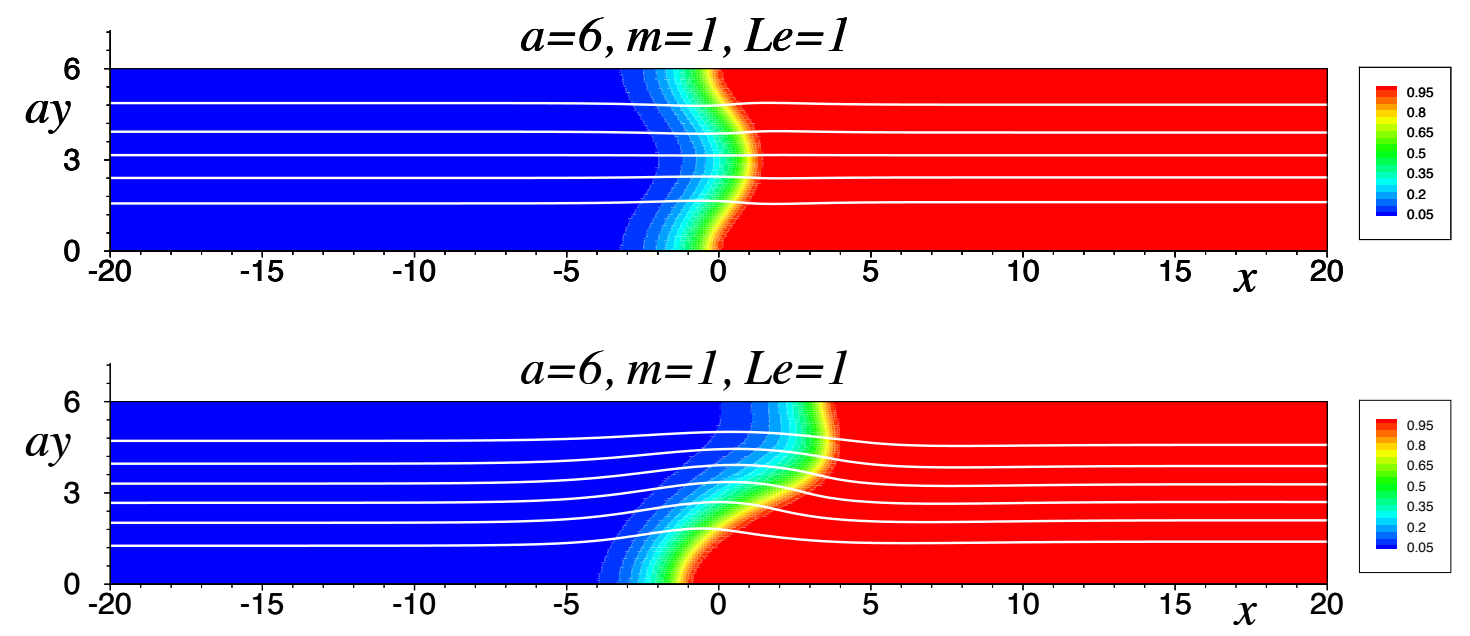

Figure 2: Symmetric (upper plot) and non-symmetric (lower plot) steady flames (color isotherms) and streamlines (white lines) in an adiabatic channel, for $a=6, m=1, L e=1$, $\beta=10, q=5, n=2$.

in narrow channel configurations. One can see that for a given channel width, $a$, non-symmetric flames appear for flow rates above a critical value, $m=m_{c}$. These bifurcation points, marked in Fig. 1 with open circles, are shown in Fig. 3 as a function of the channel width $a$. It is clear in this figure that there exists a critical channel thickness (indicated approximately with a vertical line), to the left of which only symmetric flames exist.

\subsection{Influence of thermal expansion}

The influence of thermal expansion is shown in Fig. 4, where the dimensionless velocity $u_{f}$ (left plot) and the symmetry indicator $S$ (right plot) are plotted as functions of $m$ for several values of the thermal expansion parameter $q$. All curves were calculated for $\beta=10$ and $L e=1$. The solid lines represent symmetric solutions and the dashed lines non-symmetric ones. The bifurcation points $m_{c}$ above which non-symmetric flames are possible are marked with open circles. One can see that with decreasing values of $q$ the bifurcation point shifts to the the right.

The constant density or thermo-diffusive limit widely used in combustion studies can be obtained, formally, by taking $q \rightarrow 0$ in Eqs. (3)-(8) only for a fixed Zel'dovich number, $\beta$. The necessity to fix the Zeldovich number stems from the fact that, in a strict sense, $\beta=N q /(1+q)^{2}$, where $N=E / \mathcal{R}_{g} T_{0}$. Thus, the limit $q \rightarrow 0$ has sense only for $\beta$ fixed in Eq. (9). The other possibility would be to fix both $\beta$ and $q$ in the reaction rate term Eq. (9) and to apply $q=0$ in the equation of state Eq. (8). This last choice was used in $[13,14]$. In any of the two cases, the 


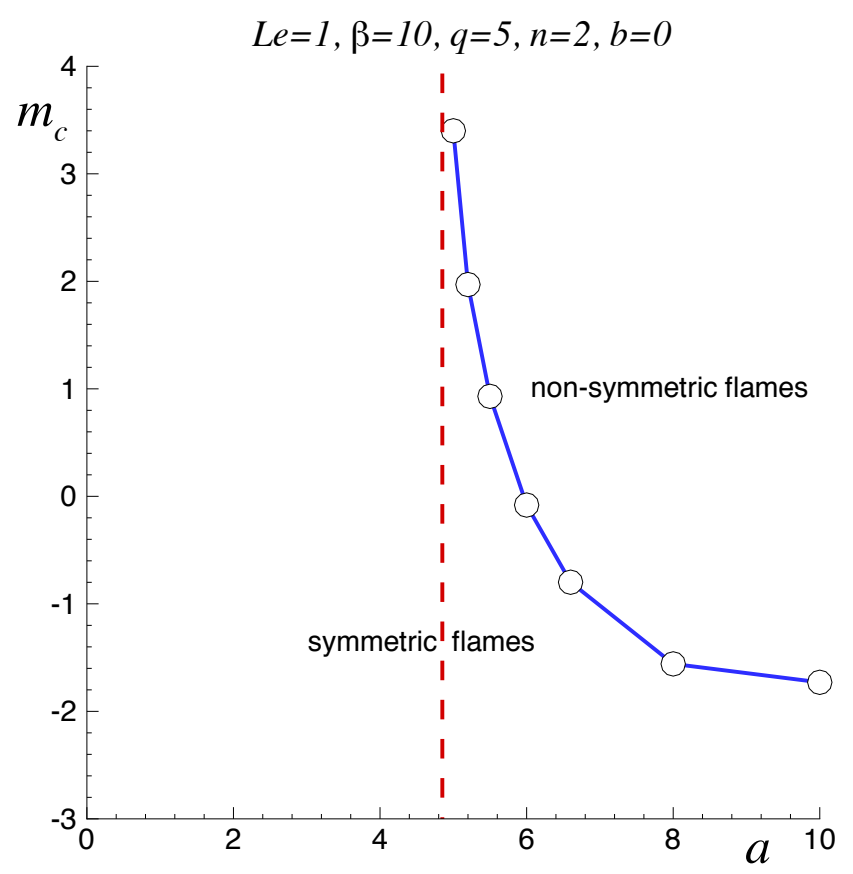

Figure 3: The bifurcation point $m_{c}$ plotted as a function of the channel width $a$ for $L e=1$, $\beta=10, q=5, n=2$ and $b=0$; the non-symmetric solutions exist above the curve; the vertical dashed line shows the approximate critical value of $a$.

system of governing equations is reduced to the temperature and mass fraction equations with a prescribed parabolic velocity profile. No non-symmetric flames were found for $L e=1$ in this limit, see $[13,14]$. When this result is compared with that of Eqs. (3)-(7), it is evident that the bifurcation value $m_{c}$ tends to infinity as $q \rightarrow 0$ for $L e=1$. This is shown in Fig. 5, where $m_{c}$ is plotted as a function of $q$ calculated for a case with $a=6, L e=1, \beta=10$ and $b=0$.

\subsection{Influence of heat-losses}

Up to this point, adiabatic channels have been considered, with $b=0$ applied in the boundary condition Eq. (10). Figure 6 shows the properties $u_{f}$ (left plot) and $S$ (right plot) as functions of $m$ several values of the heat losses parameter $b$, all curves plotted for $a=8, L e=1, \beta=10$ and $q=5$. The results indicate that the bifurcation value of the flow rate, $m_{c}$, shifts rapidly to larger values of $m$ even with a moderate increase of $b$. This agrees with the results obtained by Tsai, see [11], where channels with isothermal walls were studied and non-symmetric flames were obtained only for significantly large values of the duct width, of order $D / \delta_{T}=O(100)$, 

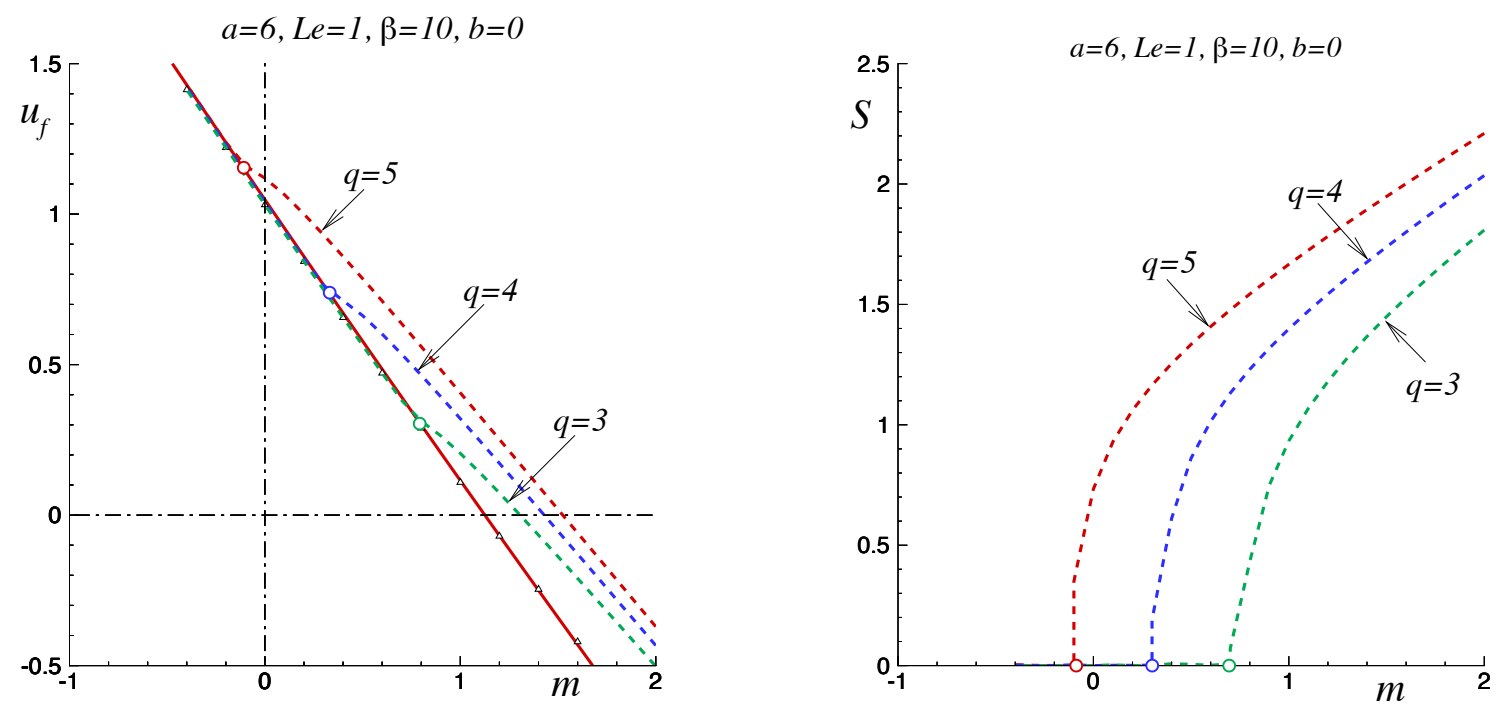

Figure 4: The computed flame velocity $u_{f}=U_{f} / S_{L}$ (left plot) and the symmetry indicator $S$ (right plot) as functions of the non-dimensional flow rate $m=U_{0} / S_{L}$ for $L e=1$ and several values of $q$. The solid line and the small triangles in the left plot correspond to symmetric flames calculated for $q=5$ and $q=3$, respectively, dashed lines to non-symmetric flames; all curves calculated for $a=6, \beta=10, n=2$ and an adiabatic channel, $b=0$; the bifurcation points are marked with open circles.

where $D$ was the diameter of the duct.

\subsection{Influence of the Lewis number}

The structure of flames at low Lewis numbers is illustrated in Fig. 7, where a symmetric and a non-symmetric flame are shown for the same set of parameters. These solutions correspond to the points marked with open triangles in Fig. 10. It is well known that for $L e<1$ there is a region behind the flame where the temperature exceeds the adiabatic flame temperature due to the combined effect of the flame curvature and differential diffusion. One can see that in the non-symmetric flame case the temperature maximum is significantly higher.

The numerical analysis carried out in [13] showed that for $L e<1$ non-symmetric flames can exist even in the framework of the constant density model, $q=0$. The incorporation of the thermal expansion effect, $q>0$, reinforces this phenomenon. Figure 8 shows the flame velocity $u_{f}$ and the symmetry indicator $S$ plotted versus $m$ for $a=6, \beta=10, q=5$ and several values of Le. Solid and dashed lines correspond to results calculated with $n=2$ while diamond symbols 


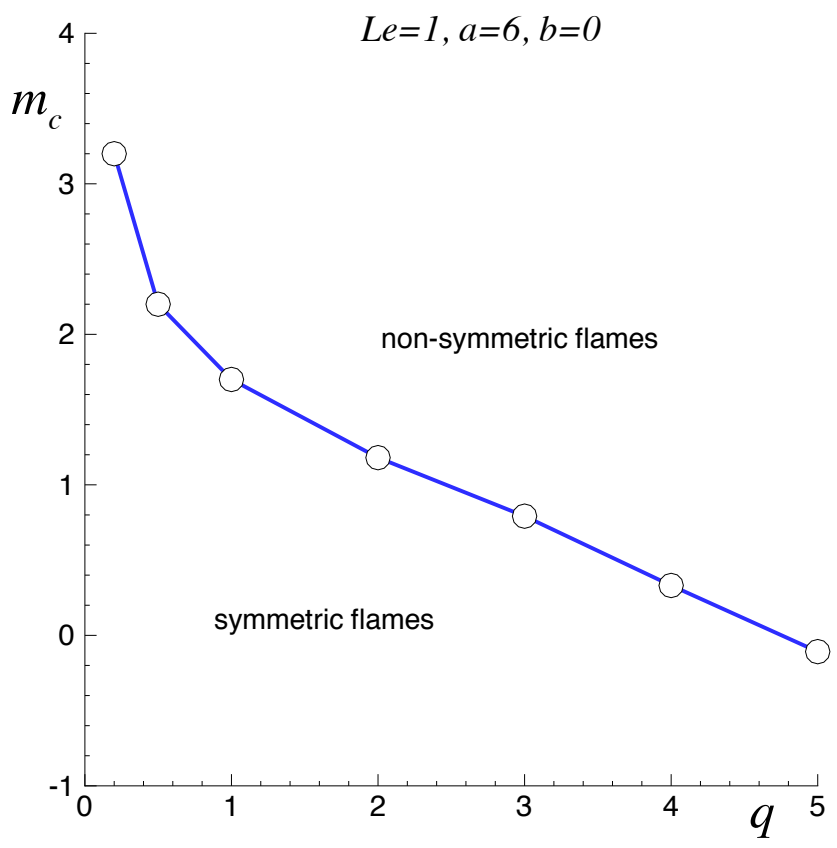

Figure 5: The bifurcation point $m_{c}$ plotted as a function of the thermal expansion parameter $q$, calculated for $a=6, L e=1, \beta=10, n=2$ and $b=0$; the non-symmetric solutions exist to the right of the curve.

represent points calculated with $n=1$ (only for $L e=0.9$ ). One can see again, as in Fig. 1, that the influence of the parameter $n$ in the flame propagation velocity or the bifurcation point is relatively small.

Figure 8 shows that with the decreasing of $L e$ below one the bifurcation point $m_{c}$ shifts to smaller (negative) values and therefore the region where non-symmetric flames appear becomes significantly wider. Notice also that non-symmetric flames are observed even for Lewis numbers larger than one, as shown in Fig. 8 for the case with $L e=1.05$. Nevertheless, it should be emphasized that as $L e$ is increased above one the critical flow rate $m_{c}$ is offset to the right, so that non-symmetric flames can exist only for very large values of the flow rate $m$. The dependence of $m_{c}$ with the Lewis number Le is given in Fig. 9 for two values of the channel width $a$ and for $\beta=10, q=5$ and $n=2$.

All bifurcations from symmetric to non-symmetric solutions described in the preceding sections and corresponding to $L e=1$ were found to be supercritical. We show now that, apparently, subcritical bifurcations occur for lower Lewis numbers. Figure 10 presents the flame velocity 

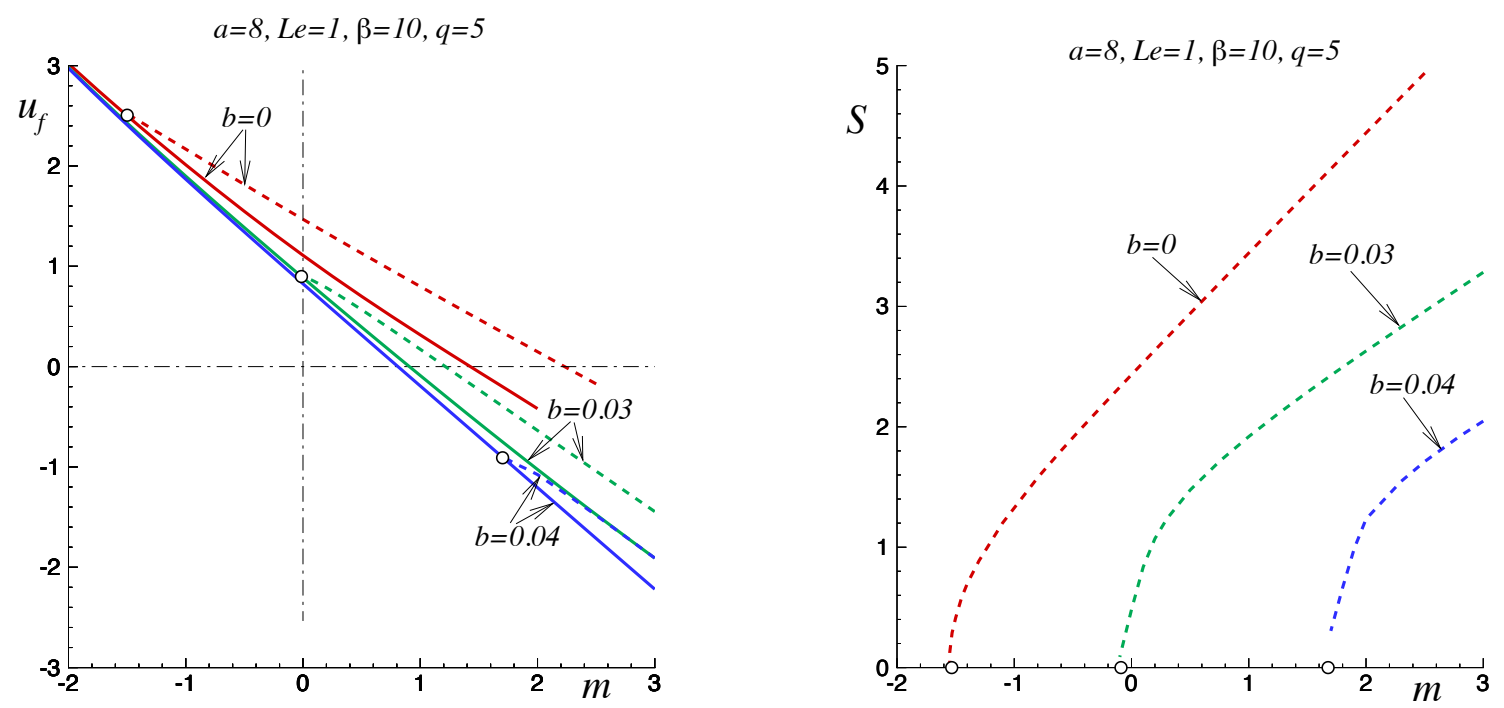

Figure 6: The computed flame velocity $u_{f}=U_{f} / S_{L}$ (left plot) and the symmetry indicator $S$ (right plot) as functions of the non-dimensional flow rate $m=U_{0} / S_{L}$ for $L e=1$ and several values of $b$. Solid lines - symmetric flames, dashed lines - non-symmetric flames; all curves calculated for $\beta=10, q=5, n=2$; the bifurcation points are marked with open circles.
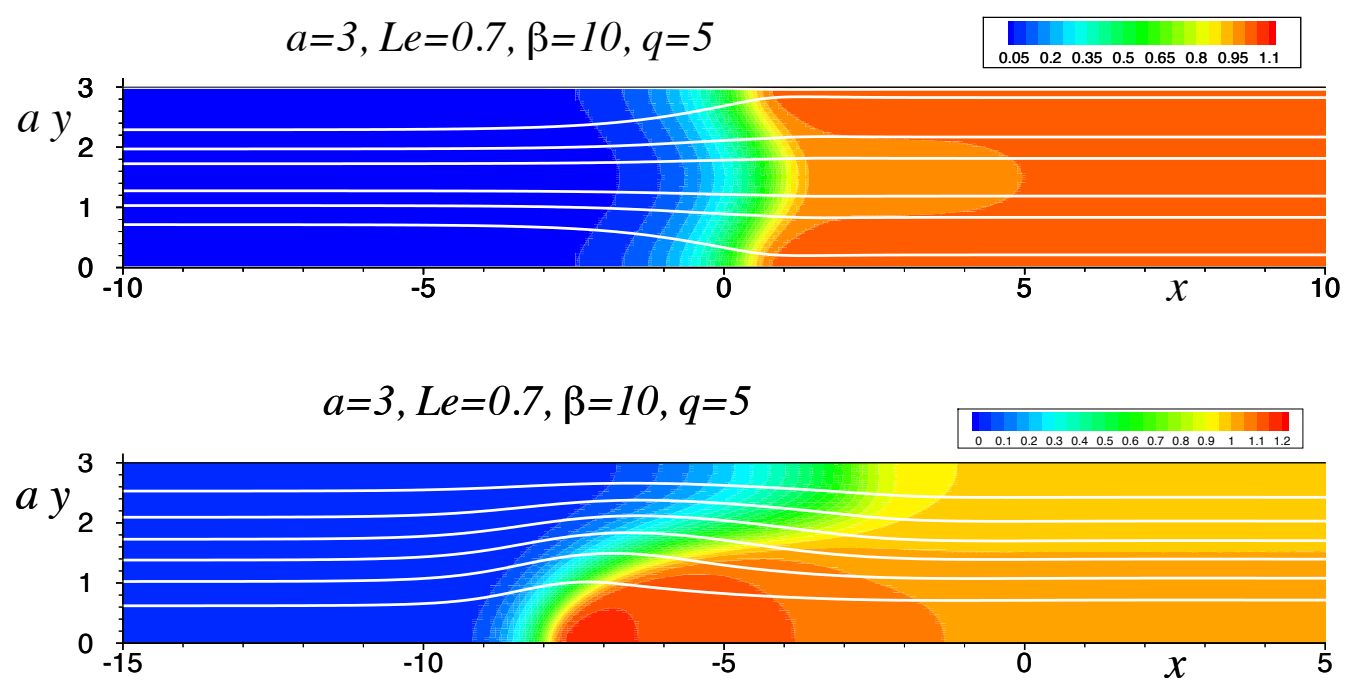

Figure 7: Symmetric (upper plot) and non-symmetric (lower plot) steady flames (color isotherms) and streamlines (white lines) in an adiabatic channel for $a=3, m=2, L e=0.7$, $\beta=10, q=5, n=2$; the states are marked in Fig. 10 with open triangles. 

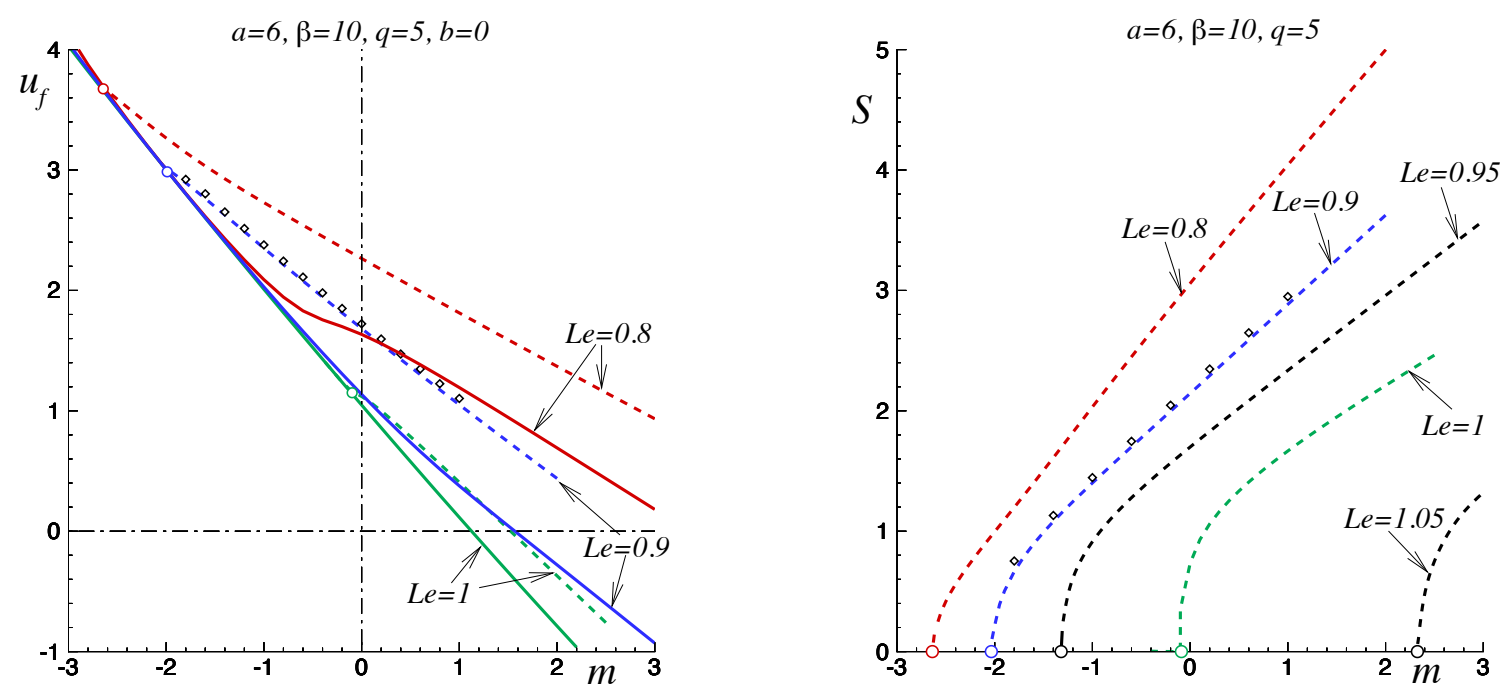

Figure 8: The computed flame velocity $u_{f}=U_{f} / S_{L}$ (left plot) and the symmetry indicator $S$ (right plot) as functions of the non-dimensional flow rate $m=U_{0} / S_{L}$ calculated for several values of $L e$ in an adiabatic channel, $b=0$ : solid and dashed lines represent symmetric and non-symmetric flames, respectively, calculated with $n=2$; diamond symbols show the results calculated with $n=1$ (only for $L e=0.9$ ); all curves calculated for $a=6, \beta=10$ and $q=5$; the bifurcation points are marked with open circles.

$u_{f}$ (left plot) and the symmetry indicator $S$ (right plot) calculated for $L e=0.7, \beta=10, q=5$, $n=2$ and several values of $a$ in adiabatic channels. The dashed-line curves represent the nonsymmetric solutions and the solid-line curves the symmetric ones. The symmetric solutions have very close values of $u_{f}$ below some value of $m$, becoming practically indistinguishable.

One can see in Fig. 10 that moving along a dashed-line curve toward lower values of $m$ the non-symmetric solution suddenly jumps to the symmetric one at the points marked with open circles, with a significant and abrupt change in $u_{f}$. It is safe to suggest that there should exist a middle branch (with certainty unstable) which connects the dashed and solid curves at some point. Unfortunately the numerical methods used in the present study did not permit to calculate this middle branch.

The conditions of upstream flame propagation, known in the literature as the flashback effect, occur for $m<m_{f l b}$, where $m_{f l b}$ is a critical point determined by $u_{f}=0$. In other words, for the flow rate $m \approx m_{f l b}$ the flame does not move (or, practically does not move) with respect to the wall. This stationary condition is very useful in experiments to visualize easily the flame 


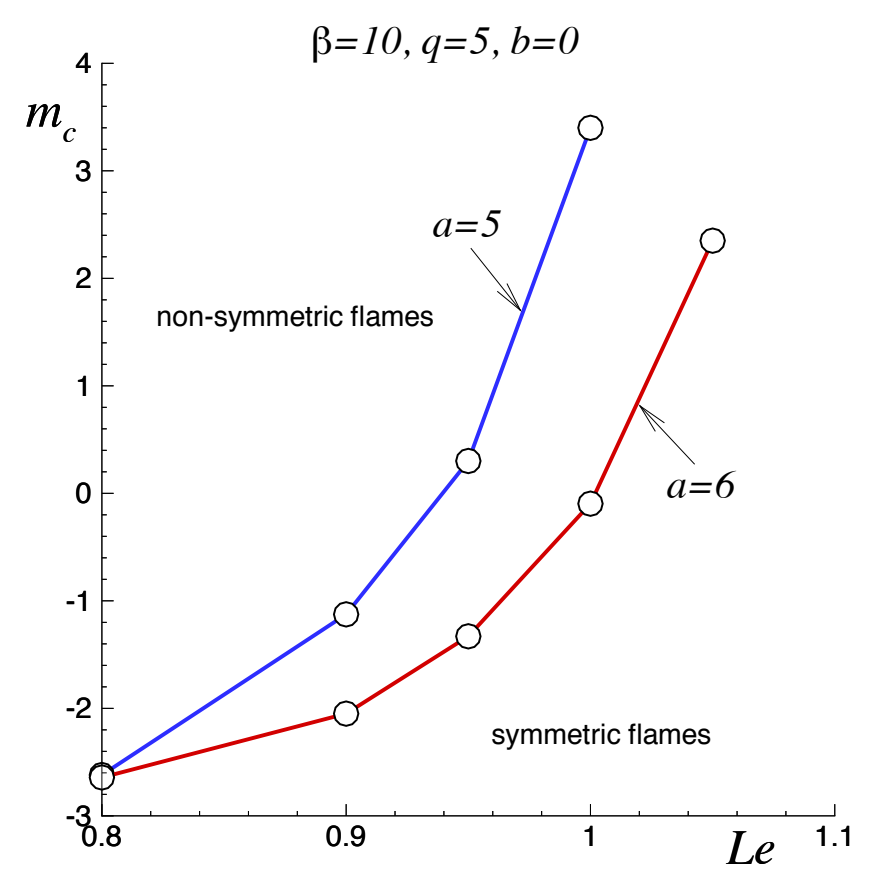

Figure 9: The bifurcation point $m_{c}$ plotted as a function of the Lewis number $L e$, calculated for $a=5$ and $6, \beta=10, q=5$ and $b=0$; the non-symmetric solutions exist above the curves.

and study its structure. It is also of great practical consequence in many combustion devices from a safety point of view. Investigation of this condition has received a notable attention in the literature, see [31] as a recent example.

Figures 1, 4, 6 and 8 put in evidebce the importance of properly taking into account the possible symmetry breaking when determining the flashback conditions. The critical flashback flow rates, $m_{f l b}$, calculated imposing symmetric conditions (that is, computed in a half channel) result to be significantly lower than those predicted when symmetry is not imposed. But in reality, the physically relevant flash-back point in Figs 1, 4, 6 and 8 is unique in the majority of cases, because when the non-symmetric solution exists for the same set of parameters, then the symmetric one is unstable.

An interesting situation can be observed in Fig. 10 for a narrow channel with $a=2.5$. One can see that moving from the right to the left along the corresponding dashed curve it crosses the axis $u_{f}=0$ at $m_{f l b} \approx 3.193$, below which $u_{f}$ becomes positive. For further decreasing in $m$, at the left of the subcritical bifurcation point marked with an open circle, the flame jumps to the solid line and becomes symmetric, propagating then with negative $u_{f}$. This line crosses 

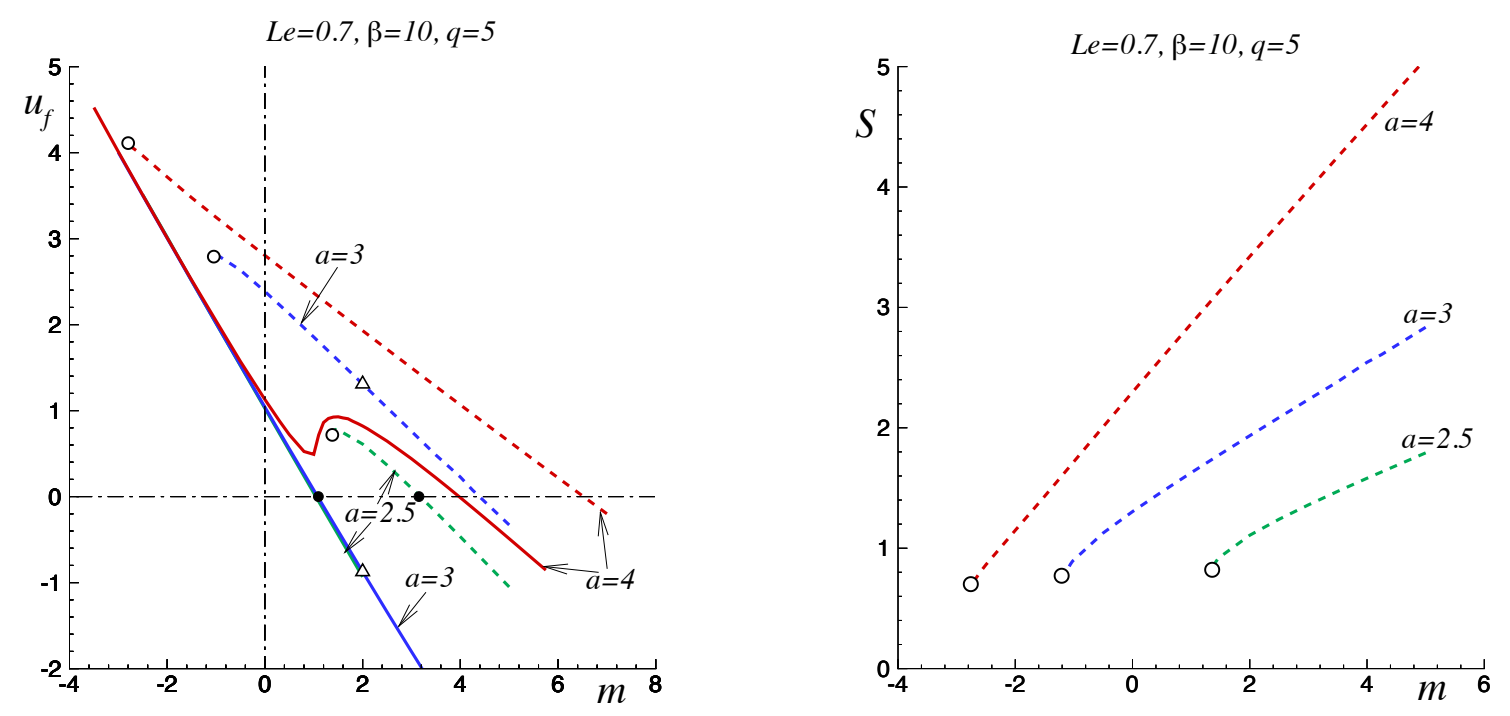

Figure 10: Computed flame velocity $u_{f}=U_{f} / S_{L}$ (left plot) and the symmetry indicator $S$ (right plot) as functions of $m=U_{0} / S_{L}$ calculated for several values of $a$ illustrating subcritical bifurcations for $L e=0.7, \beta=10, q=5$ and $b=0$ : for $m$ below the open circles nonsymmetric solutions exhibit a jump to symmetric ones; solid lines - symmetric flames, dashed lines - non-symmetric flames. Two (stable) flash-back points are marked with dark circles.

again the axis $u_{f}=0$ at $m_{f l b} \approx 1.033$. These two flash-back points are indicated in Fig. 10 with black circles and the corresponding temperature, reaction rate, mass fraction and streamlines are shown in Fig. 11. Both of these flash-back solutions, one non-symmetric and one symmetric, are stable, as may be inferred from the results of unsteady simulations presented in the next section.

\section{Unsteady results}

The use of the diffusive-thermal model or the diffusive-thermal model used in [13, 14] provided a significant advantage in order to get a clear physical insight on the stability properties of symmetric and non-symmetric solutions, because the simplification in hydrodynamics made global stability analysis feasible. In the general case of variable density the difficulties in the stability analysis become formidable. This is why in the present study the stability of the solutions is determined by carrying out direct numerical time-dependent simulations.

Figure 12 shows the time histories of the flame velocity, $u_{f}$, and the symmetry indicator, $S$, calculated for the flow rates $m=1$ and $m=-1$ in the channel with $a=6$ width, all curves 

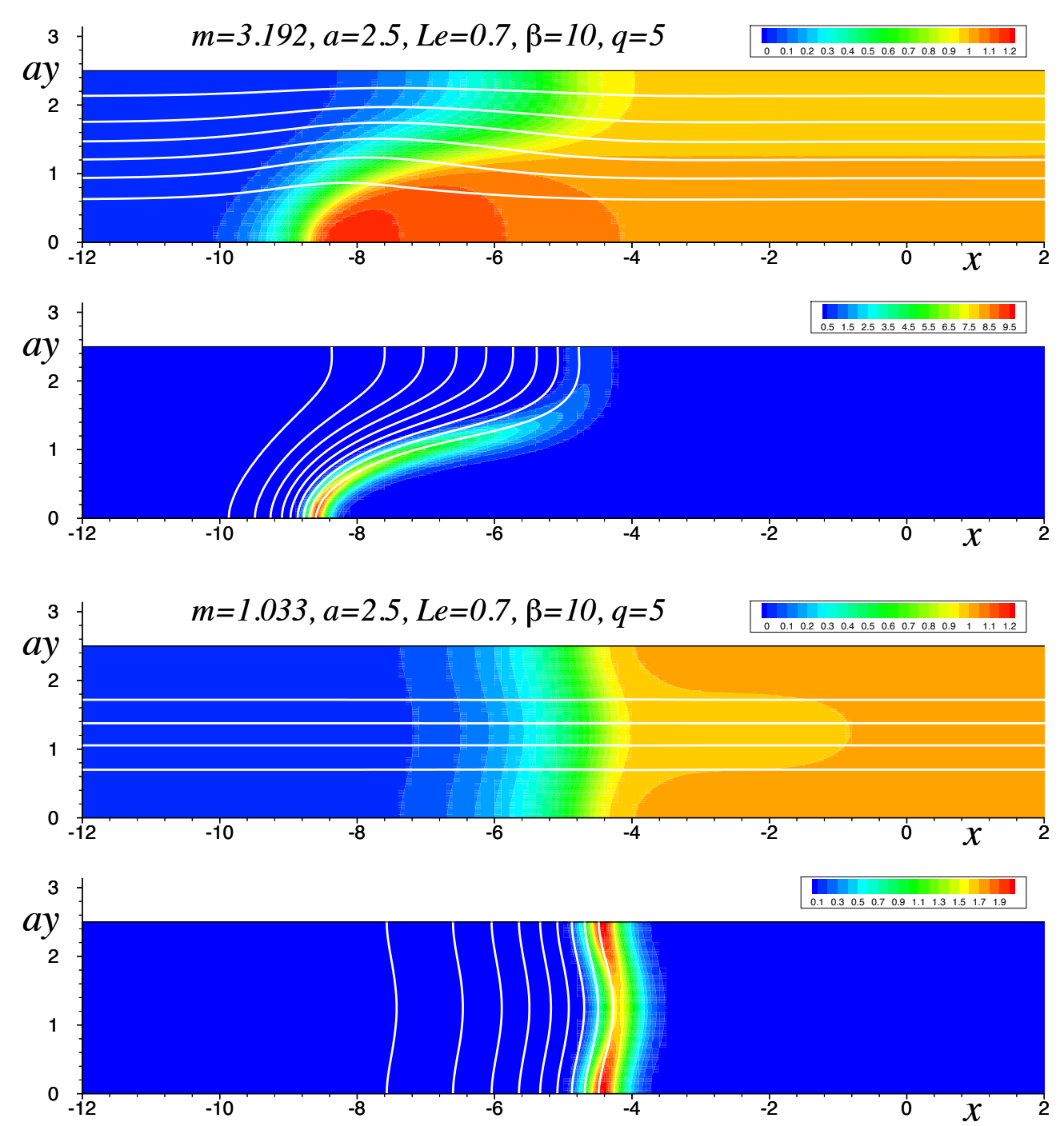

Figure 11: Structure of flames computed at the flash-back points (black circles in Fig. 10) for $a=6, L e=0.7, \beta=10, q=5$. Upper plots: non-symmetric flame. Lower plots: symmetric flame For each solution: color isotherms and isolines of stream function (white lines); color reaction iso-levels and mass fraction isolines (white lines).

obtained for $\beta=10$ and $q=5$. For the case $m=1$, when symmetric and non-symmetric flames exist simultaneously (see Fig. 1), two types of initial conditions were used. The first initial conditions, corresponding to a solid curve in Fig. 1, were visibly non-symmetric and the second initial conditions, corresponding to a dashed curve in Fig. 1, were approximately symmetric with weak perturbations. One can see that for both types of initial conditions the solutions approach a non-symmetric profile $(S \neq 0)$ after a transient time, showing therefore that the stable solution corresponding to the flow rate $m=1$ is the non-symmetric flame. It should be noted that the 

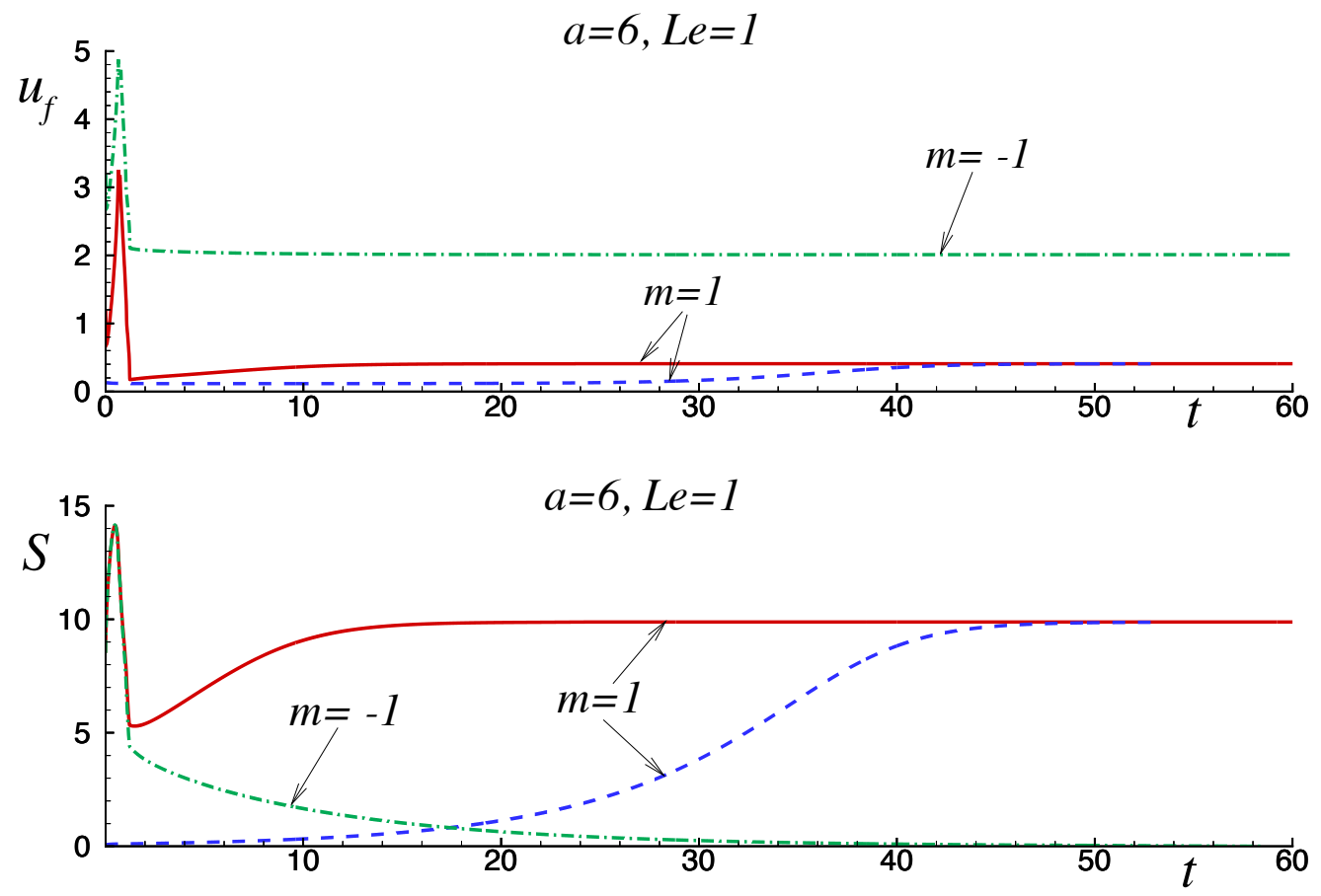

Figure 12: Time history of the flame velocity $u_{f}$ and symmetry indicator $S$, calculated for $a=6$, $L e=1, \beta=10, q=5$ and $n=2$; non-symmetric initial conditions with $m=1$ - a solid line; symmetric initial conditions weakly perturbed with $m=1$ - a dashed line; non-symmetric initial condition with $m=-1$ - a dash-dotted line.

intensity of the weak perturbations applied in the second case does affect the transient time and for extremely weak perturbations this time may be very long. Obviously this time depends also on the intrinsic accuracy (e.g the numerical mesh size) of the code.

For the case $m=-1$ shown in Fig. 12 only a symmetric solution exists. The initial conditions applied in this case were non-symmetric and the time dependent evolution, indicated with a dash-dotted line, brought the flame to a symmetric profile $(S \approx 0)$.

Similar patterns were observed for other sets of parameters. These time-dependent results confirm those reported in $[13,14,16]$ obtained by global stability analysis: when the nonsymmetric flames exist simultaneously with symmetric ones, the non-symmetric flames are stable and the symmetric flames are unstable.

Figure 13 illustrates the unsteady behavior near the multiple flash-back points found for $a=2.5, L e=0.7, \beta=10, q=5$ and $n=2$ (see Fig. 10) with a plot of the time history of the dimensionless velocity, $u_{f}$. One can see that, starting from different initial conditions and imposing values of $m$ close to the flash-back values, the flame evolves to the states with $u_{f} \approx 0$ in both cases. 


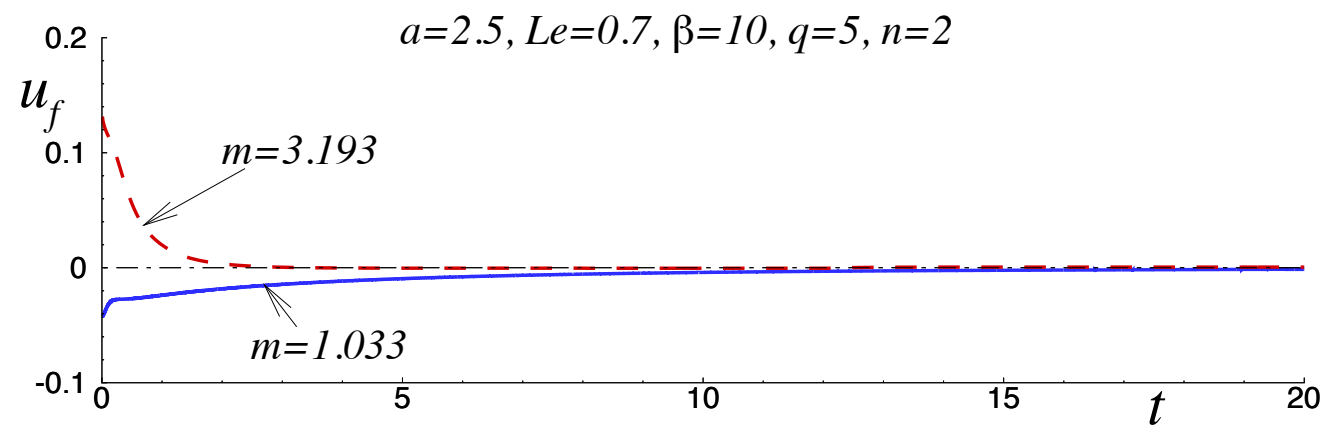

Figure 13: Time history of the flame velocity $u_{f}$ illustrating stability of the two flashback states marked with black circles in Fig. 10 (left plot): $m=3.193$ - a dashed line (non-symmetric flame), $m=1.033$ - a solid line (symmetric flame)

These results of time dependent simulations permit, firstly, to validate the conclusion that the two flash-back states shown in Fig.10 are stable and therefore both can be physically realized. Secondly, they indicate that the flame can be stabilized with respect to the wall at two different flow rates, which is an important warning for the design of experiments.

\section{Conclusions}

Even if the existence of non-symmetric solutions in the free propagation of flames in narrow channels is apparently well known, since non-symmetric flame shapes have been shown in various publications, little attention has been paid to the determination of the critical conditions for their emergence. The case in point is the situation when both the governing equations and the corresponding boundary conditions are symmetric about the midplane in two-dimensional channels or axisymmetrically invariant in the cases of circular channels but, in spite of this fact, the flame shape is not symmetric. The present investigation tries to throw light upon this phenomenon.

The exception is the studies carried out in the frame of the constant density model. In this case the hydrodynamic part of the governing equations is simplified, reducing the flow field to a parabolic Poiseuille velocity profile not affected by the flame. These studies revealed that for a certain parametric range symmetric and non-symmetric solutions coexist. Besides, when this is the case, the symmetric solutions are unstable. This fact was established by means of global stability analysis, facilitated significantly by the constant density simplifications.

Within the constant density framework only symmetric solutions were found if the Lewis 
number was exactly equal to one. Nevertheless, it was demonstrated that for Lewis numbers below one, even just slightly below, there is always a critical channel width above which the non-symmetric solutions appear, see [13].

The results of the present work show that the incorporation of thermal expansion into the problem greatly enriches the phenomenon of flame symmetry-breaking. We have found that even for $L e=1$ the flame becomes non-symmetric in adiabatic channels with widths and flow rates above some critical values, $a_{c}$ and $m_{c}$, respectively.

We have also shown that as the parameter governing thermal expansion, $q$, decreases, the critical flow rate, $m_{c}$, above which non-symmetric flames appear, shifts to larger values. Presumably, this critical flow rate, $m_{c}$, tends to infinity as $q \rightarrow 0$, in agreement with the results obtained in the framework of the constant density model.

Summing up the above results one can conclude that at least two different kinds of instabilities/effects contribute simultaneously to the emergence of non-symmetric flames in narrow channels. The first one is the differential-diffusion instability and the second is the Darrieus-Landau instability. Fortunately, they can be switched off/on considering the constant-density model (the Darrieus-Landau instability is suppressed) or the unity Lewis number case (the differentialdiffusion instability is absent). Obviously these phenomena can not be separated in the general case of variable density and $L e<1$.

Our results also show that the emergence of non-symmetric flames is notably sensitive to heat losses. As a general trend one can conclude that the increase in heat losses brings stability to the symmetric flame. All these results affect the flash-back conditions or, what is equivalent, the reactant flow rate for which the flame is stationary with respect to the wall, a condition often studied in experiments on flame propagation in ducts.

Results of numerical calculations carried out for $L e<1$ are also aligned with those obtained within the constant density model. We found that the critical channel width above which nonsymmetric flames exist decreases as $L e$ decreases. It was also demonstrated that for a sufficiently low Lewis number the symmetric/non-symmetric bifurcation becomes subcritical. In particular, a case with multiple flash-back conditions, one corresponding to a symmetric flame and the other to with a non-symmetric flame, was found. This example highlights the difficulties that can appear in experiments, where the flash-back critical condition is often the preferred operating point in order to study the flame structure in channels. 


\section{Acknowledgments}

The present work is supported by project \#ENE2015-65852-C2-2-R ( MINECO / FEDER, EU).

\section{References}

[1] S. T. Lee, J. S. Tien, A numerical analysis of flame flashback in a premixed laminar system, Combust. Flame 48 (1982) 273-285.

[2] S. T. Lee, C. H. Tsai, Numerical investigation of steady laminar flame propagation in a circular tube, Combust. Flame 99 (1994) 484-490.

[3] J. Daou, M. Matalon, Flame propagation in Poiseuille flow under adiabatic conditions, Combust. Flame 124 (2001) 337-349.

[4] V. N. Kurdyumov, E. Fernández-Tarrazo, Lewis number effect on the propagation of premixed laminar flames in narrow open ducts, Combust. Flame 128 (2002) 382-394.

[5] J. Daou, M. Matalon, Influence of conductive heat-losses on the propagation of premixed flames in channels, Combust. Flame 128 (2002) 321-339.

[6] C. Cui, M. Matalon, J. Daou, J. Dold, Effects of differential diffusion on thin and thick flames propagating in channels, Combust. Theory Model. 8 (2004) 41-64.

[7] C. Cui, M. Matalon, T. Jackson, Pulsating mode of flame propagation in two-dimensional channels, AIAA Journal 4 (2005) 1284-1292.

[8] V. Gamezo, E. Oran, Flame acceleration in narrow tubes: effect of wall temperature on propulsion characteristics, AIAA paper 2006-1134.

[9] V. Akkerman, V. Bychkov, A. Petchenko, L.-E. Eriksson, Accelerating flames in cylindrical tubes with nonslip at the walls, Combust. Flame 145 (2006) 206-219.

[10] A. Petchenko, V. Bychkov, V. Akkerman, L.-E. Eriksson, Flamesound interaction in tubes with nonslip walls, Combust, Flame 149 (2007) 418-434.

[11] C. H. Tsai, The asymmetric behavior of steady laminar flame propagation in ducts, Combust. Sci. Tech. 180 (2008) 533-545.

[12] M. Short, D. Kessler, Asymptotic and numerical study of variable-density premixed flame propagation in a narrow channel, J. Fluid Mech. 638 (2009) 305-337. 
[13] V. N. Kurdyumov, Lewis number effect on the propagation of premixed flames in narrow adiabatic channels: Symmetric and non-symmetric flames and their linear stability analysis, Combust. Flame 158 (2011) 1307-1317.

[14] V. Kurdyumov, C. Jiménez, Propagation of symmetric and non-symmetric premixed flames in narrow channels: influence of conductive heat-losses, Combust. Flame 161 (2014) 927936.

[15] D. Fernández-Galisteo, C. Jiménez, M. Sánchez-Sanz, V. N. Kurdyumov, The differential diffusion effect of the intermediate species on the stability of premixed flames propagating in microchannels, Combust. Theory Model. 18 (2014) 582-605.

[16] V.N. Kurdyumov, C, Jiménez, Structure and stability of premixed flames propagating in narrow channels of circular cross-section: Non-axisymmetric, pulsating and rotating flames, Combust. Flame 167 (2016) 149-163.

[17] A. Dejoan, V. Kurdyumov, Thermal expansion effect on the propagation of premixed flames in narrow channels of circular cross-section: Mulriplicity of solutions, axisymmetry and non-axisymmetry., Proc. Combust. Inst. 37 (2019) 1927-1935.

[18] V. N. Kurdyumov, M. Matalon, Flame acceleration in long narrow open channels, Proc. Combust. Inst. 34 (2013) 865-872.

[19] V. N. Kurdyumov, M. Matalon, Self-accelerating flames in long narrow open channels, Proc. Combust. Inst. 35 (2015) 921-928.

[20] C. Jiménez, D. Fernández-Galisteo, V. N. Kurdyumov, DNS study of the propagation and flashback conditions of lean hydrogen-air flames in narrow channels: Symmetric and nonsymmetric solutions, Int. J. Hydrogen Energy 40 (2015) 12541-12549.

[21] C. Jiménez, V. Kurdyumov, Propagation of symmetrc and non-symmetric lean hydrogenair flames in narrow channels: Influence of heat losses, Proc. Combust. Inst. 36 (2017) 1559-1567.

[22] N.I. Kim, T. Kataoka, Sh. Maruyama, K. Maruta, Flammability limits of stationary flames in tubes at low pressure, Combust. Flame 141 (2005) 78-88.

[23] H. Pearlman, Excitability in high-Lewis number premixed gas combustion, Combust. Flame 109 (1997) 382-398. 
[24] U. Dogwiler, J. Mantzaras, P. Benz, B. Kaeppeli, R. Bombach, A. Arnold, Homogeneous ignition of methane-air mixtures over platinum: Comparison of measurements and detailed numerical predictions, Proc. Combust. Inst. 27 (1998) 2275-2282.

[25] G. Pizza, C. Frouzakis, J. Mantzaras, A. Tomboulide, K. Boulouchos, Dynamics of premixed hydrogen/air flames in microchannels, Combust. Flame 152 (2008) 433-450.

[26] G. Pizza, C. Frouzakis, J. Mantzaras, A. Tomboulides, K. Boulouchos, Dynamics of premixed hydrogen/air flames in mesoscale channels, Combust. Flame 155 (2008) 2-20.

[27] G. Pizza, J. Mantzaras, C. Frouzakis, A. Tomboulides, K. Boulouchos, Suppression of combustion instabilities of premixed hydrogen-air flames in microchannels using heterogeneous reactions, Proc. Combust. Inst. 32 (2009) 3051-3058.

[28] E. Miyata, N. Fukushima, Y. Naka, M. Shimura, M. Tanahashia, T. Miyauchi, Direct numerical simulation of micro combustion in a narrow circular channel with a detailed kinetic mechanism, Proc. Combust. Inst. 35 (2015) 3421-3427.

[29] V. N. Kurdyumov, G. Pizza, C. E. Frouzakis, J. Mantzaras, Dynamics of premixed flames in a narrow channel with a step-wise wall temperature, Combust. Flame 156 (2009) 21902200.

[30] K. Bioche, A. Pieyre, G. Ribert, F. Richecoeur, L. Vervisch, The role of gravity in the asymmetry of flames in narrow combustion chambers, Combust. Flame 203 (2019) 238246.

[31] K. Bioche, L. Vervisch, G. Ribert, Premixed fame-wall interaction in a narrow channel: impact of wall thermal conductivity and heat losses, J. Fluid Mech. 856 (2018) 5-35.

[32] http://www.openfoam.com. 